\title{
Two-Phase Swirling Flow in a Gas-Liquid Separator*
}

\author{
Hironobu KATAOKA ${ }^{* *}$, Akio TOMIYAMA ${ }^{* *}$, Shigeo HOSOKAWA ${ }^{* *}$, \\ Akira $\mathrm{SOU}^{* *}$ and Masao $\mathrm{CHAKI}^{* * *}$ \\ **Graduate School of Engineering, Kobe University, \\ Rokkodai 1-1, Nada, Kobe, 657-8501, Japan \\ E-mail: tomiyama@mech.kobe-u.ac.jp \\ *** Power and Industrial Systems R \& D Lab, Hitachi Ltd., \\ Omika 7-2-1, Hitachi, 319-1221, Japan \\ E-mail: masao.chaki.dx@hitachi.com
}

\begin{abstract}
Air-water swirling flows in a one-fifth model of a steam separator in a boiling water nuclear reactor are measured to obtain a database for modeling and verification of numerical methods for predicting swirling flows in the separator. Flow patterns, liquid film thicknesses, separated flow rates and the ratio $W_{s}{ }^{*}$ of the separated flow to the total liquid flow are measured using a high-speed camera, a laser focus displacement meter and flowmeters. Main conclusions obtained are as follows: (1) liquid transfer from droplets to liquid film is caused not only by droplet deposition but also by the collection of droplets on the vanes of the swirler, (2) $W_{s}{ }^{*}$ increases with the gas volume flux $J_{G}$ and does not depend on the liquid volume flux $J_{L}$ so much because a large centrifugal force caused by the swirler makes most of droplets in the gas core deposit on the liquid film before the separation and (3) a local peak appears in the axial distribution of film thickness, the position of which corresponds to the location where the droplet deposition caused by the centrifugal force has completed.
\end{abstract}

Key words: Gas-Liquid Two-Phase Flow, Steam Separator, Annular Flow, Swirling Flow, Film Thickness, Boiling Water Reactor

\section{Introduction}

Boiling water nuclear reactors, BWR, are equipped with steam separators for splitting a two-phase mixture into steam and water before feeding steam to dryers and turbines. The steam separator consists of a standpipe, a diffuser with a swirler, and a barrel with several pick-off-rings (POR). Stationary vanes of the swirler apply a large centrifugal force to the flow inside the barrel, by which most of water rapidly migrates toward the barrel wall. An annular swirling flow with few droplets in the gas core is, therefore, to be formed in the barrel. The liquid film flow is separated from the gas core flow by making use of flow passages formed by the gap between the pick-off-ring and the barrel.

Sufficient knowledge on annular swirling flow in a vertical pipe and numerical methods for predicting the flow are definitely indispensable for rational design and improvement of the steam separators. Though Wakasugi et al. ${ }^{(1)}$ and Yoneda $\&$ Inada $^{(2)}$ measured swirling two-phase flows in separators, the gas flow rates tested were so low that no data were taken for annular swirling flows. Nakao et al. ${ }^{(3)}$ and Ikeda et al. ${ }^{(4)}$ measured pressure drops and rates of liquid separation. However no detailed information on annular swirling flows has been reported yet.

Air-water swirling flows in a downscaled model of the steam separator are, therefore, 
measured in this study to obtain an experimental database applicable to the modeling and verification of numerical methods for predicting swirling flows in the separator. Flow patterns, liquid film thicknesses, flow rates of separated flows and the rate of liquid separation are measured using a high-speed video camera, a Laser focus displacement meter and flowmeters, respectively. The role played by the swirler in separation is examined by conducting experiments with and without the swirler. In addition, two types of PORs are used to study the effects of gap size between the barrel and POR on the performance of liquid separation.

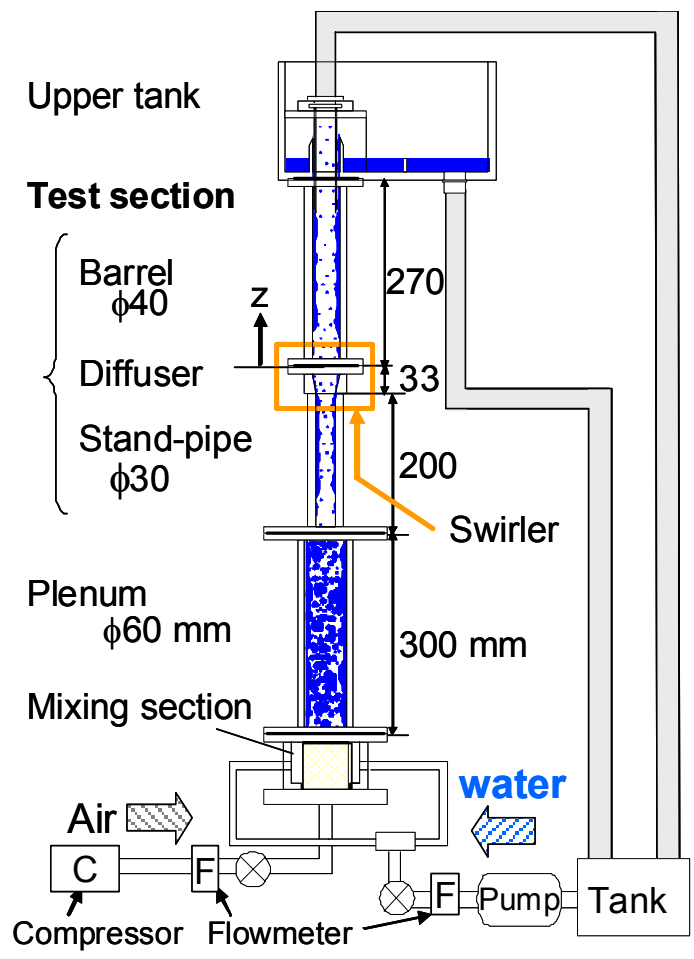

Fig. 1 Experimental apparatus

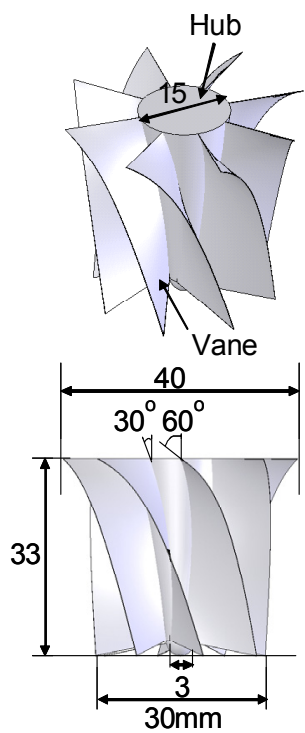

Fig. 2 Swirler

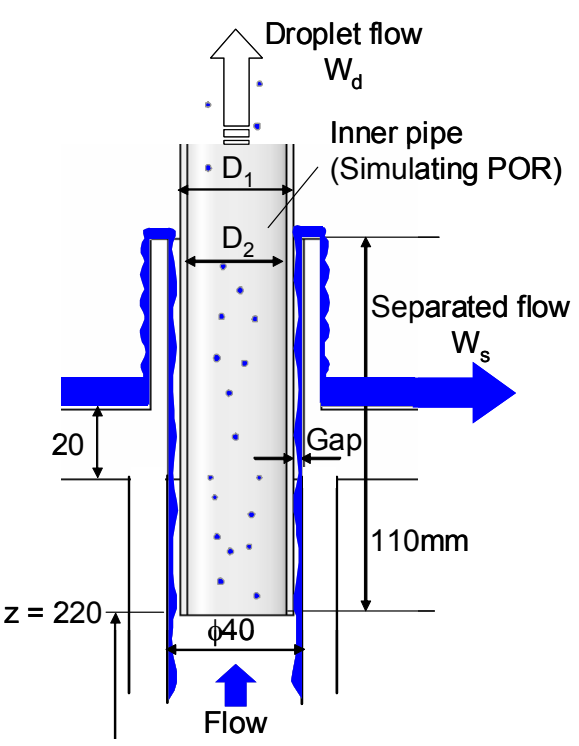

Fig. 3 Pick-Off-Ring (POR) 
Table 1 Inner pipes for simulating POR

\begin{tabular}{c|ccc}
\hline & $\mathrm{D}_{1}[\mathrm{~mm}]$ & $\mathrm{D}_{2}[\mathrm{~mm}]$ & Gap [mm] \\
\hline Type 1 & 32 & 28 & 4 \\
Type 2 & 36 & 32 & 2 \\
\hline
\end{tabular}

\section{Experimental}

Figure 1 shows the experimental apparatus. It consisted of the upper tank, the test section for simulating the steam separator, the plenum, the gas-liquid mixing section, the water supply system and the air supply system. The test section consisted of the barrel, the diffuser and the standpipe, and was made of transparent acrylic resin for observation and optical measurements of two-phase flows. The size was one-fifth of the actual steam separator used in BWR. Air was supplied from the oil-free compressor (Oil-free Scroll 11, Hitachi Ltd.), the regulator (R600-20, CKD) and the flowmeter (FLT-N, Flowcell, Ltd.) to the mixing section. Tap water at room temperature $\left(20^{\circ} \mathrm{C}\right)$ was supplied from the magnet pump (MD-40RX Iwaki, Ltd.) and the flowmeter to the mixing section. Air was introduced in the chamber of the mixing section from the bottom, while water was introduced through the wall of the chamber made of porous-sinter so as to form a uniform azimuthal distribution of the liquid flow in the mixing section. The two-phase flow formed in the mixing section flowed up through the plenum of $60 \mathrm{~mm}$ in inner diameter $D$ and $300 \mathrm{~mm}$ in length $L$, the standpipe of $D=30 \mathrm{~mm}$ and $L=200 \mathrm{~mm}$, the diffuser of $L=33 \mathrm{~mm}$ and the barrel of $D=40 \mathrm{~mm}$ and $L=270 \mathrm{~mm}$.

The swirler shown in Fig. 2, which was made of ABS resin, was installed in the diffuser to form a swirling flow in the barrel. Its shape was based on an actual swirler. Experiments without the swirler were also conducted to examine the role played by the swirling flow in flow characteristics and the performance of the separator. As will be discussed later, most of flow patterns observed in the barrel were annular flows consisting of liquid film flow, gas flow and droplet flow.

Figure 3 shows the details of the upper part of the test section including the upper tank and the device for separating the liquid film flow from the gas core flow, i.e. the mixture of gas and droplet flows. In an actual steam separator, the so-called pick-off-ring, POR, is utilized for the separation. The inner pipe of $2 \mathrm{~mm}$ in thickness was inserted in the barrel to simulate POR. The lower end of the inner pipe located $220 \mathrm{~mm}$ above the bottom of the barrel. Two types of inner pipes shown in Table 1 were used to study the effects of the gap size on the separation performance. Most of the liquid film flowed through the gap between the barrel wall and the outer wall of the inner pipe, while most of air and droplets flowed through the inner pipe. The separated liquid and the droplets carried over returned to the water reservoir through independent pipelines.

Experimental conditions were determined by adjusting the values of the flow quality $x$ and the two-phase centrifugal force $F_{C}$ in the experiments to cover those in the nominal operating condition of the BWR separator. The quality $x$ and the centrifugal force $F_{C}$ were evaluated using

$$
\begin{gathered}
x=\frac{\rho_{G} J_{G}}{\rho_{G} J_{G}+\rho_{L} J_{L}} \\
F_{C}=\frac{\rho_{m}\left(J_{G}+J_{L}\right)^{2}}{R}
\end{gathered}
$$

where the subscripts $G$ and $L$ denote the gas and liquid phases, $\rho$ is the density, $J$ the area-averaged volume flux in the barrel, the subscript $m$ the gas-liquid mixture and $R$ the radius of the barrel. The mixture density $\rho_{m}$ was estimated using the following simple 
homogeneous model:

$$
\begin{gathered}
\rho_{m}=\rho_{G} \alpha+\rho_{L}(1-\alpha) \\
\alpha=\frac{x \rho_{L}}{x\left(\rho_{L}-\rho_{G}\right)+\rho_{G}}
\end{gathered}
$$

As a result of the above consideration on the experimental conditions, the values of $x, J_{G}$ and $J_{L}$ corresponding to the nominal operating condition were determined as $x=0.18, J_{G}$ $=14.6 \mathrm{~m} / \mathrm{s}$ and $J_{L}=0.08 \mathrm{~m} / \mathrm{s}$. Hence the present experiments were carried out under the conditions of $J_{G}=4.0-24.1 \mathrm{~m} / \mathrm{s}$ and $J_{L}=0.02-0.14 \mathrm{~m} / \mathrm{s}$.

Flow patterns in the barrel and the standpipe were observed using a high-speed video camera (Redlake Motion Pro HS-1, frame rate $=2000-2500$ frame/s, exposure time $=100$ $\mu \mathrm{s})$. The mass flow rates $W_{s}$ and $W_{d}$ of separated and droplet flows in the gas core were measured at the reservoir using a timer and a graduated cylinder. Each measurement was conducted for 50 seconds to make the uncertainty in measured flow rates less than $3 \%$. The ratio $W_{s}^{*}$ of the separated flow rate to the total liquid flow rate,

$$
W_{s}^{*}=\frac{W_{s}}{W_{s}+W_{d}},
$$

was used as an index of the separator performance.

The film thickness $\delta$ was measured using a laser focus displacement meter (LFD, LT-9030, Keyence, Ltd.) ${ }^{(5)}$. The sampling period was $0.64 \mathrm{~ms}$ and the measurement time was 30 seconds. The sampling number was, therefore, more than 46000 points, the number of which was sufficient to obtain accurate time-averaged film thicknesses. The uncertainty in measured $\delta$ was $2.0 \%{ }^{(5)}$.

\section{Results and Discussion}

\subsection{Flow pattern}

Recorded images of flow patterns without and with the swirler are shown in Figs. 4 and 5 , respectively. In both cases, the flow pattern transits from churn to annular flows as $J_{G}$ increases. Under the churn flow conditions, the liquid film sometimes falls along the barrel wall, whereas under the annular flow conditions the film continuously flows up. Without the swirler, the droplet deposition takes place all over the barrel. In the case of swirling flow, the rotational speed of the flow increases with $J_{G}$, and as shown in Fig. 5 (c) spiral streaks are formed from the swirler vanes in the annular flow condition. Most of the liquid in the streak is made up of liquid deposited on the swirler vanes, that is, the liquid transfer from droplets to the film is caused not only by the direct droplet deposition but also by the collection of droplets on the vanes ${ }^{(6)}$. As for the direct deposition, most of droplets deposit on the liquid film within a short distance from the swirler (about $150 \mathrm{~mm}$ ) due to a large centrifugal force generated by the swirling flow. This, in turn, implies that few droplets remain in the gas core flow in far downstream of the swirler. Though it is not clear from still images, an example of the droplet deposition captured as spots on the film, which were formed by the impact of droplets, is shown in Fig. 6 .

Figure 7 shows a comparison between the flow pattern transition model proposed by Mishima \& Ishii $^{(7)}$ and measured flow patterns in the barrel and standpipe. As can be understood from Figs. 7 (a) and (b), the value of $J_{G}$ at the transition from churn to annular flows is lower in swirling flows than in non-swirling flows. This is due to the rapid accumulation of liquid on the film caused by the large centrifugal force. Even without the swirler, the transition occurs at $J_{G}$ lower than that of the Mishima-Ishii's model. This is due 


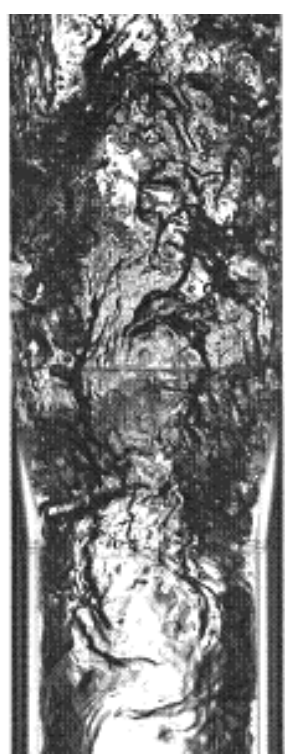

(a) Churn flow $J_{G}=5.8 \mathrm{~m} / \mathrm{s}$

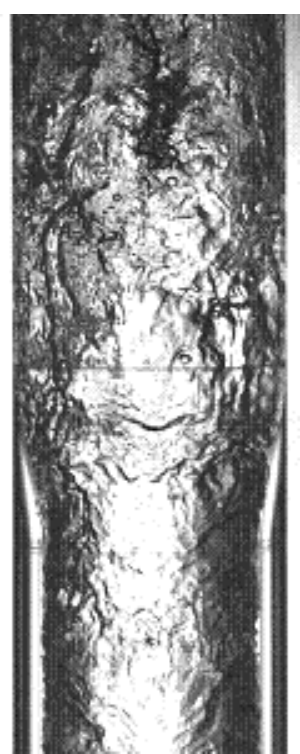

(b) Churn to annular $J_{G}=10.4 \mathrm{~m} / \mathrm{s}$

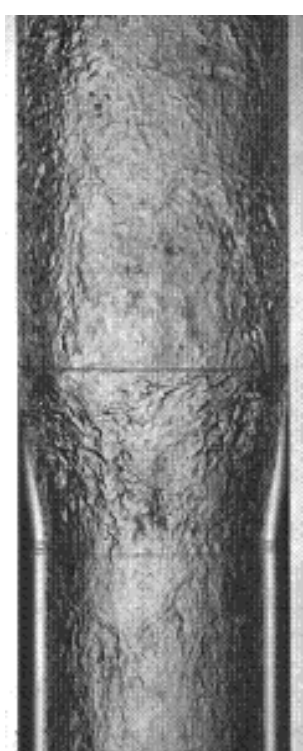

(c) Annular flow $J_{G}=17.7 \mathrm{~m} / \mathrm{s}$

Fig. 4 Flow pattern without the swirler $\left(J_{L}=0.08 \mathrm{~m} / \mathrm{s}\right)$

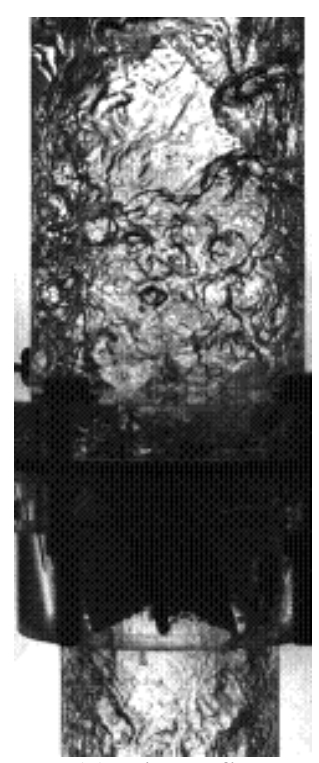

(a) Churn flow $J_{G}=5.8 \mathrm{~m} / \mathrm{s}$

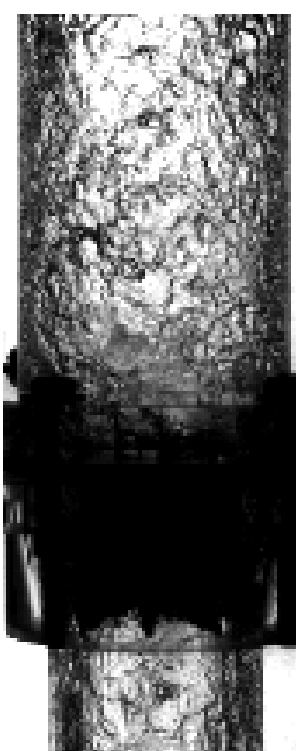

(b) Churn to annular $J_{G}=9.4 \mathrm{~m} / \mathrm{s}$

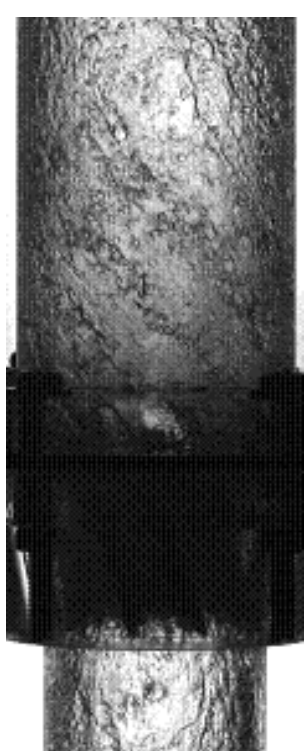

(c) Annular flow $J_{G}=17.7 \mathrm{~m} / \mathrm{s}$

Fig. 5 Flow pattern with the swirler $\left(J_{L}=0.08 \mathrm{~m} / \mathrm{s}\right)$

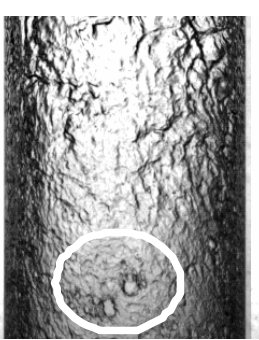

$t=0.0 \mathrm{~ms}$

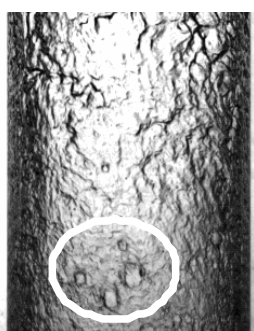

$t=2.0 \mathrm{~ms}$

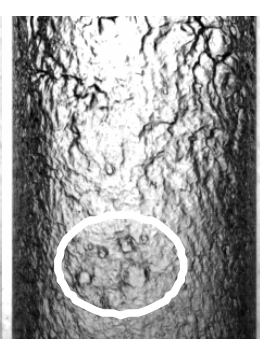

$t=4.0 \mathrm{~ms}$

Fig. 6 Spots caused by droplet deposition in the barrel $\left(J_{G}=14.6 \mathrm{~m} / \mathrm{s}, J_{L}=0.08 \mathrm{~m} / \mathrm{s}\right)$ 


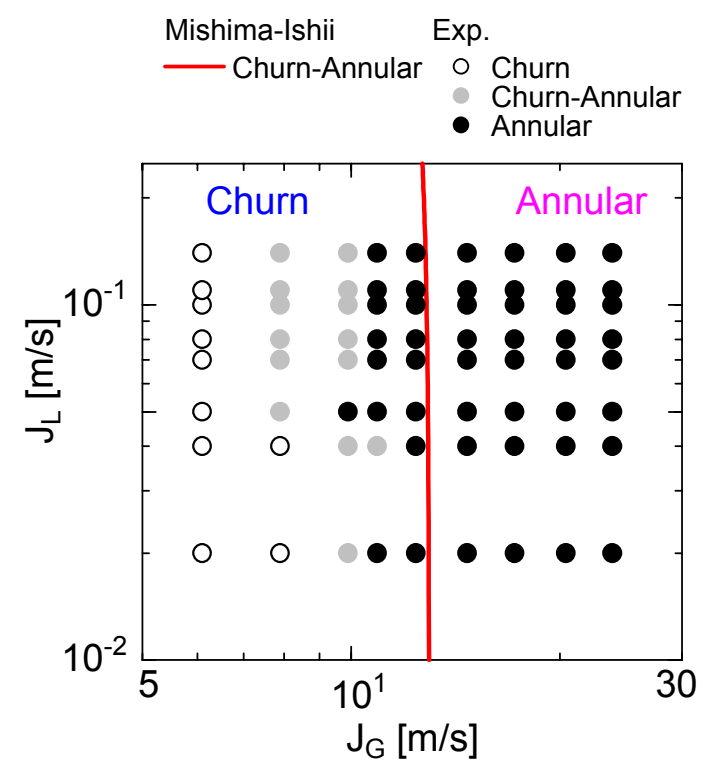

(a) Barrel (with the swirler)

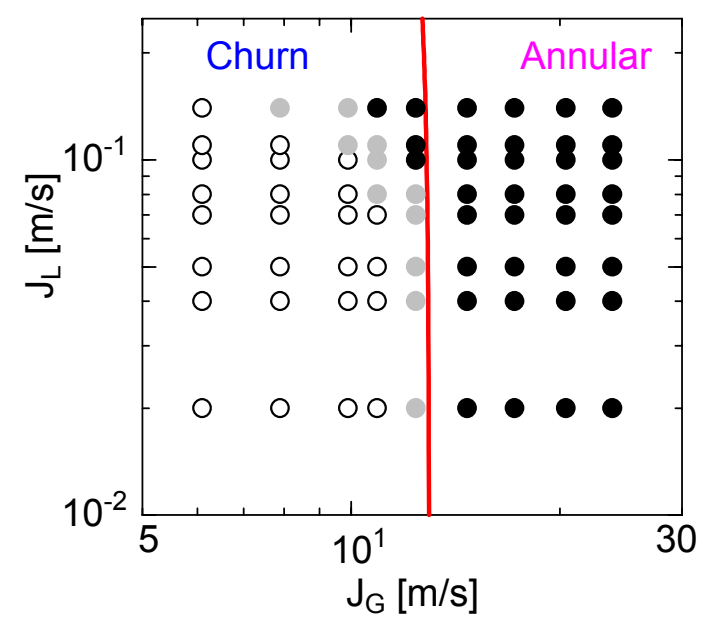

(b) Barrel (without the swirler)

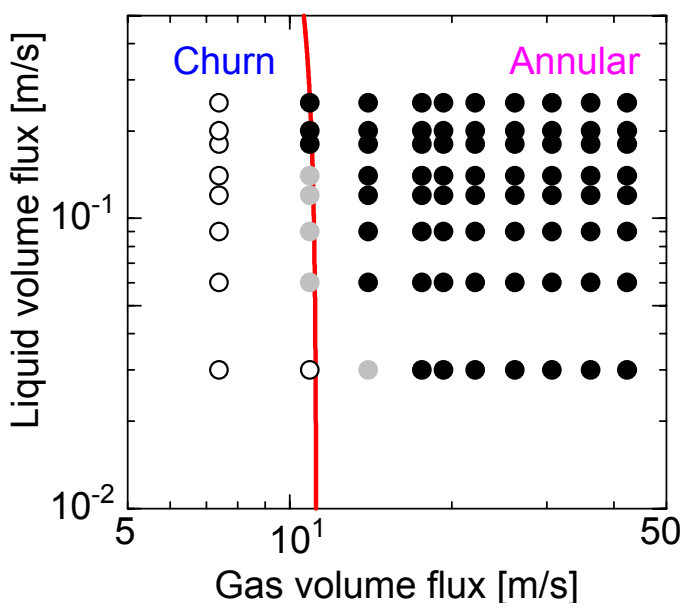

(c) Standpipe

Fig. 7 Flow pattern maps (Since $J_{G}$ and $J_{L}$ are defined by using the flow area of the barrel, $J_{G}$ and $J_{L}$ are not used in (c).) 
to the memory effect of flow pattern in upstream of the barrel. The flow pattern in the standpipe could be annular even when the flow pattern in the barrel is churn because the gas volume flux in the standpipe is larger than that in the barrel due to the smaller pipe diameter. The flow pattern in the standpipe would survive even after passing through the diffuser. This is the reason why the transition occurs at $J_{G}$ lower than that of the Mishima-Ishii's model. As shown in Fig. 7 (c), the flow pattern in the standpipe is well predicted by the Mishima-Ishii's model.

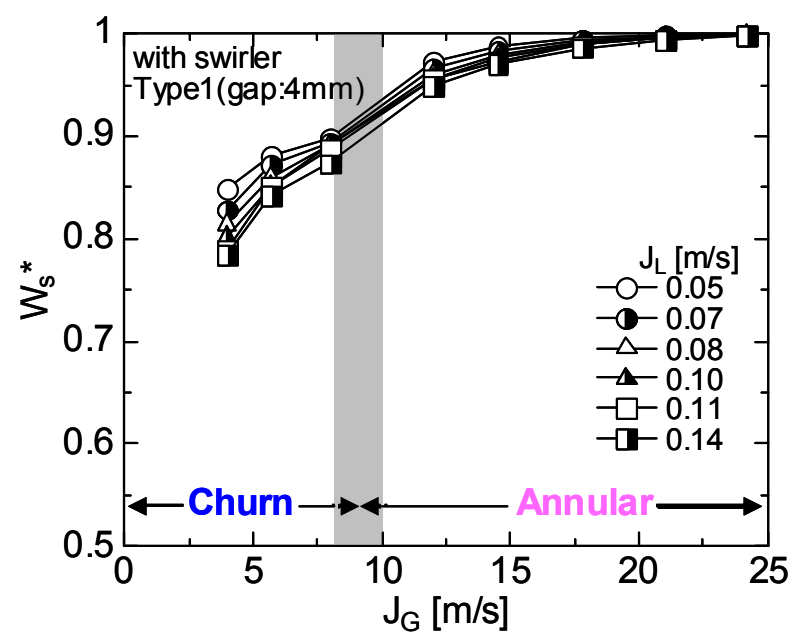

Fig. $8 W_{s}^{*}$ for swirling flows with type 1 POR

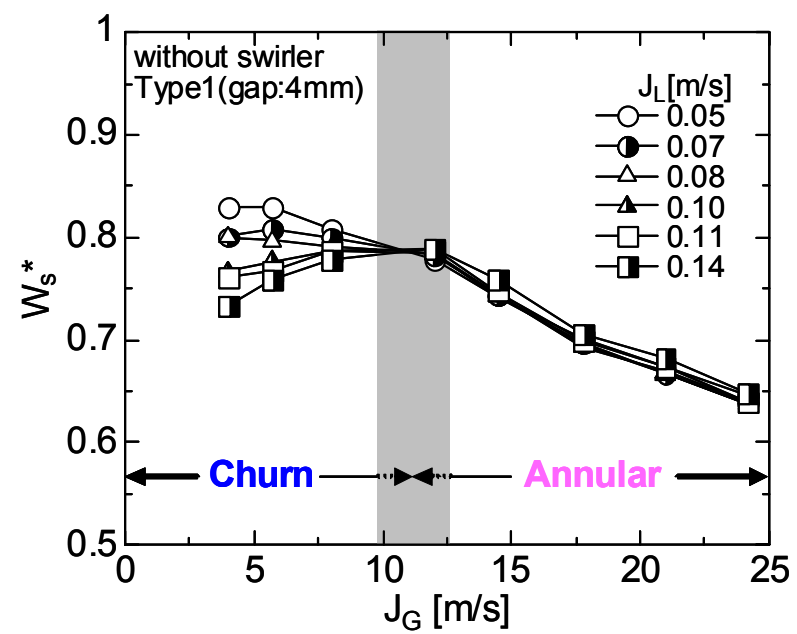

Fig. $9 W_{s}^{*}$ for non-swirling flows with type 1 POR

\subsection{Flow separation}

Figures 8 and 9 show measured $W_{s}^{*}$ for type 1 POR (gap size $=4 \mathrm{~mm}$ ) in swirling and non-swirling flows, respectively. Under churn flow conditions, (1) $W_{s}^{*}$ in both cases is not so high because of a low centrifugal force and the presence of large drops and liquid lumps in the gas core, and (2) $W_{s}^{*}$ decreases with increasing $J_{L}$ due to the increase in the number density of drops.

In swirling flows (Fig. 8), $W_{s}^{*}$ gradually increases with $J_{G}$ and asymptotically approaches unity for $J_{G}>17 \mathrm{~m} / \mathrm{s}$. This is due to the increase in the liquid film flow rate resulting from a large deposition rate caused by the increase in the centrifugal force. In annular flows, $W_{s}^{*}$ does not depend on $J_{L}$. This indicates that the droplet flow rate is negligible and all the liquid film enters into the gap at POR. 


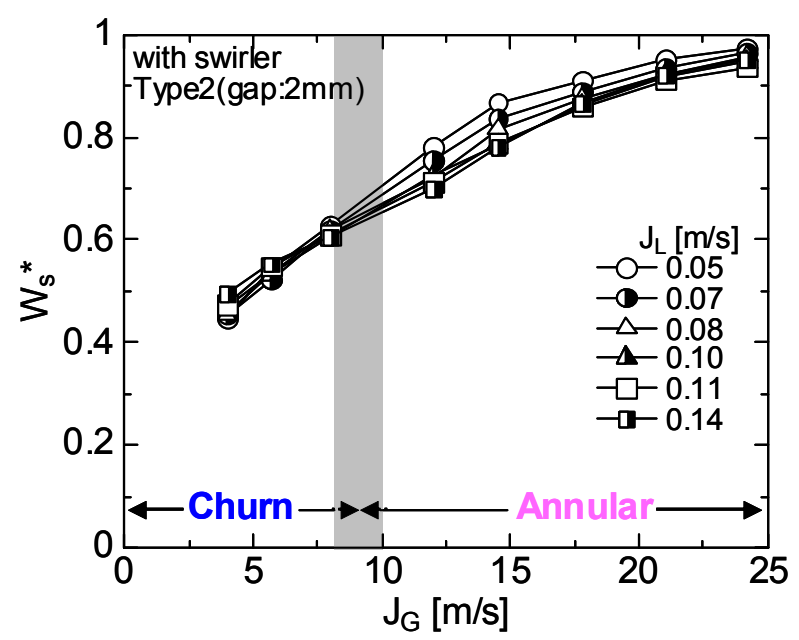

Fig. $10 W_{s}^{*}$ for swirling flows with type 2 POR

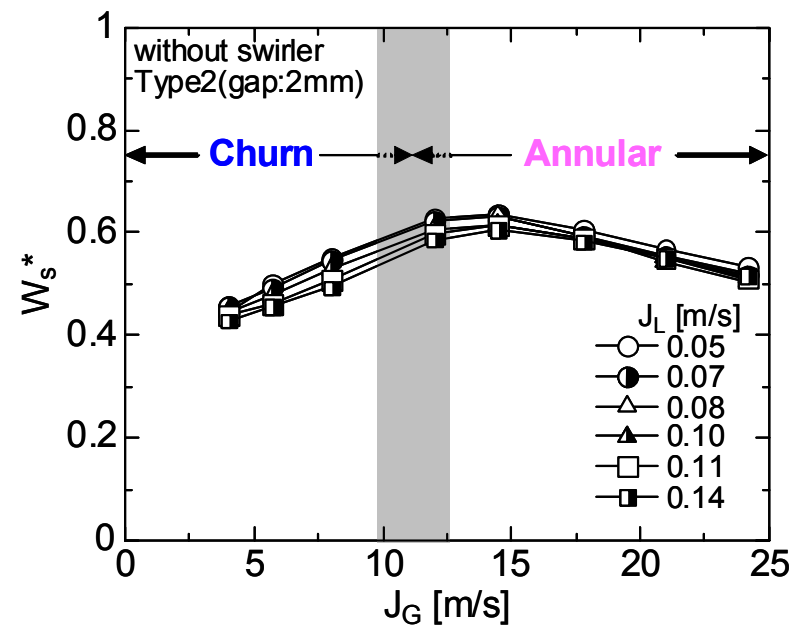

Fig. $11 W_{s}^{*}$ for non-swirling flows with type 2 POR

In non-swirling flows (Fig. 9), except for high $J_{L}$ and low $J_{G}$ conditions, $W_{s}^{*}$ gradually decreases with increasing $J_{G}$. This is because the entrainment rate increases with $J_{G}$. For the high $J_{L}$ and low $J_{G}$ conditions, the intermittency of churn flow is strong enough to form large liquid lumps, which block the cross section of the barrel and generate a number of drops. This might be the reason why $W_{s}^{*}$ is very low in these conditions.

These results elucidate the fact that the swirler is a very effective device for the flow separation. It also indicates that with the swirler, the droplet deposition increases with $J_{G}$, whereas without the swirler the droplet entrainment increases with $J_{G}$. The latter tendency is common in an ordinary annular flow in a straight pipe.

Figures 10 and 11 are measured $W_{s}^{*}$ for type 2 POR (gap size $=2 \mathrm{~mm}$ ) in swirling and non-swirling flows, respectively.

In swirling flows, being similar to type $1 \mathrm{POR}, W_{s}^{*}$ increases with $J_{G}$. However, $W_{s}^{*}$ is not close to unity even under high $J_{G}$ conditions. This might be because a part of the liquid film cannot enter into the narrow gap of type 2 POR and floods into the inner pipe. Figure 10 also indicates that $W_{s}^{*}$ depends on $J_{L}$ in annular flow conditions, i.e., for $J_{G}>11 \mathrm{~m} / \mathrm{s}, W_{s}^{*}$ decreases with increasing $J_{L}$. This decrease is caused by the increase in the liquid volume flooding into the inner pipe. 
As shown in Fig. 11, $W_{s}^{*}$ for non-swirling flows with type 2 POR shows the same trend as that with type 1 POR except for low $J_{G}$ and low $J_{L}$ conditions. That is, $W_{s}^{*}$ gradually increases with $J_{G}$ under churn flow conditions even at low $J_{G}$ and low $J_{L}$ conditions. Though a portion of the liquid film flooding into the inner pipe exists in all the flow conditions, its amount would decrease with increasing $J_{G}$ because the film thickness becomes smaller as $J_{G}$ increases. Hence the trend in the low $J_{G}$ and low $J_{L}$ conditions in type 2 POR differs from that in type 1 POR.

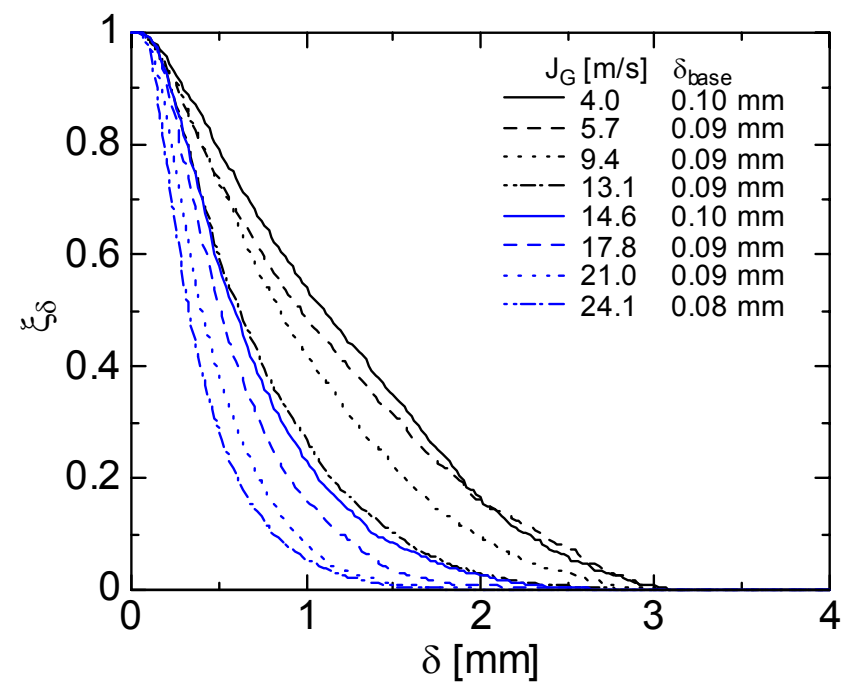

(a) Swirling flows

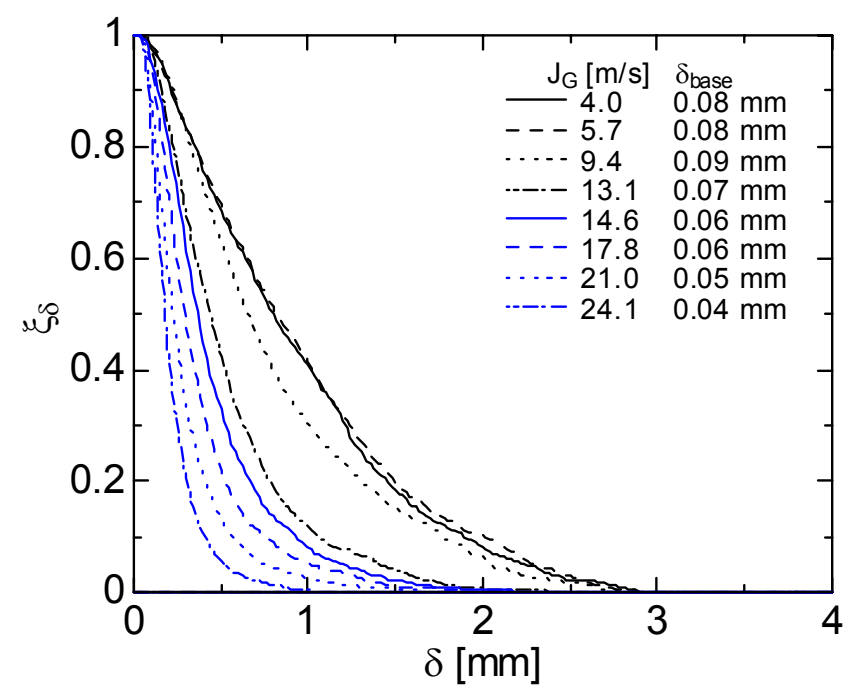

(b) Non-swirling flows

Fig. $12 \xi_{\delta}$ distributions for $J_{L}=0.08 \mathrm{~m} / \mathrm{s}$ measured at $z=170 \mathrm{~mm}$

\subsection{Film thickness}

Cumulative probability distributions $\xi_{\delta}$ of film thickness $\delta$ measured by using LFD are used to calculate the mean and base film thicknesses, $\delta_{\text {avg }}$ and $\delta_{\text {base }}$, respectively. They are defined as the arithmetic average of $\delta$ and the value of $\delta$ at $\xi_{\delta}=0.99$, respectively ${ }^{(8)}$. Discussion on the maximum film thickness will be reported in elsewhere.

Figure 12 shows $\xi_{\delta}$ distributions for $J_{L}=0.08 \mathrm{~m} / \mathrm{s}$ measured at $z=170 \mathrm{~mm}$, where $z$ is the axial distance from the diffuser ( $50 \mathrm{~mm}$ below POR). As can be understood from the values of base film thicknesses listed in the figure, $\delta_{\text {base }}$ is larger in swirling flow than in non-swirling flow. 
Axial distributions of $\delta_{a v g}$ measured at $J_{L}=0.08 \mathrm{~m} / \mathrm{s}$ are shown in Fig. 13. In swirling flows, the swirl-induced deposition causes a large increase in $\delta_{\text {avg }}$ for $z \leq 150 \mathrm{~mm}$, whereas $\delta_{\text {avg }}$ gradually decreases for $z>150 \mathrm{~mm}$. The latter is because (a) the deposition rate there is extremely low due to the low droplet concentration and (b) even a slight increase in $\delta_{\text {avg }}$ will result in a large increase in the interfacial shear stress ${ }^{(9)}$, which, in turn, causes the acceleration of film velocity and the decrease in $\delta_{a v g}$. The presence of the peak in film thickness should be taken account of when designing the axial location of POR in BWR separators.

Up to $z \sim 100 \mathrm{~mm}, \delta_{\text {avg }}$ is smaller in swirling flows than in non-swirling flows. This is because in just downstream of the swirler, the presence of the hub increases the gas velocity near the barrel wall. In non-swirling flows, $\delta_{\text {avg }}$ slightly decreases in the flow direction, which implies that the entrainment rate is larger than the deposition rate, as is often the case with ordinary annular pipe flows.

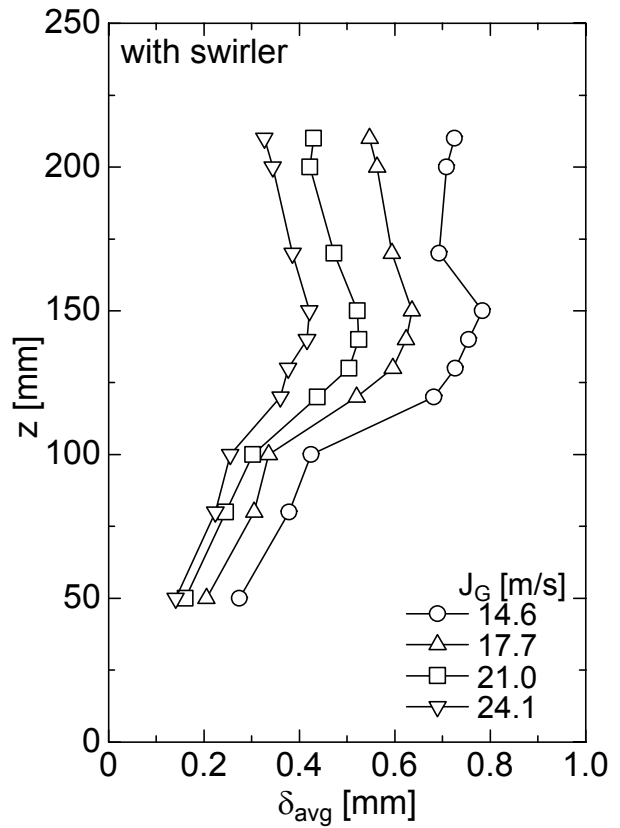

(a) Swirling flow

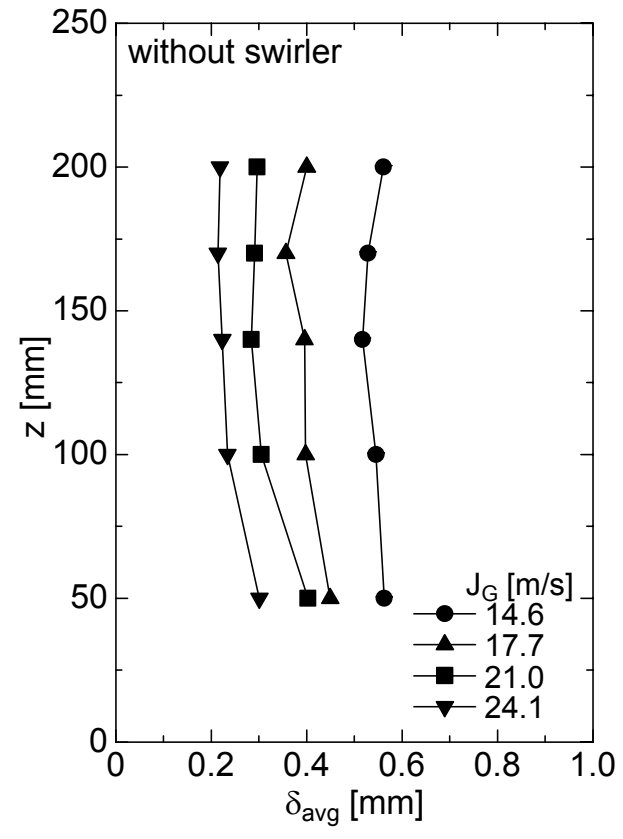

(b) Non-swirling flow

Fig. 13 Axial distributions of $\delta_{a v g}\left(J_{L}=0.08 \mathrm{~m} / \mathrm{s}\right)$

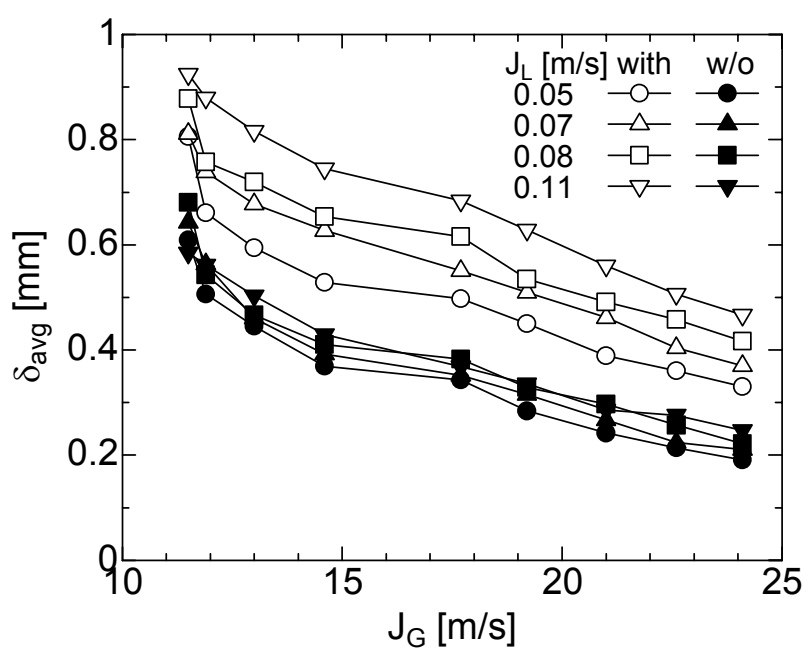

Fig. 14 Effects of $J_{G}$ and $J_{L}$ on $\delta_{a v g}(z=170 \mathrm{~mm})$ 
Measured mean film thicknesses at $z=170 \mathrm{~mm}$ are shown in Fig. 14, which clearly shows that $\delta_{\text {avg }}$ in swirling flows takes a higher value and depends more strongly on $J_{L}$ than in non-swirling flows. The strong dependence on $J_{L}$ is in accordance with the fact that the separated flow rate is close to the total liquid flow rate in swirling flows, i.e., the increase in $J_{L}$ directly reflects in the increase in the film flow rate. On the other hand, $\delta_{a v g}$ in non-swirling flows does not depend on $J_{L}$ so much. This trend is different from an ordinary annular pipe flow, in which $\delta_{\text {avg }}$ more clearly increases with $J_{L}$. This discrepancy can be explained by postulating that droplet formation in the diffuser section increases with $J_{L}$. Further study on the droplet formation at the diffuser is, however, required to confirm this postulation, though we have observed in non-swirling flows that lots of droplets are formed at the diffuser due to the strong flow agitation associated with the deceleration of flow velocity.

\section{Conclusion}

Air-water two-phase swirling flows in a downscaled model of a steam separator in a boiling water nuclear reactor are visualized using a high-speed video camera. Measurements of the liquid film thickness and separated flow rate are also carried out to obtain an experimental database which can be utilized for the development of a numerical method for predicting two-phase flows in the steam separator. The role played by a swirler in the separation is examined by conducting experiments with and without the swirler. As a result, the following conclusions are obtained.

(1) Liquid transfer from droplets to liquid film is caused not only by droplet deposition but also by the collection of droplets on the vanes of the swirler.

(2) In an annular swirling flow, the ratio $W_{s}{ }^{*}$ of the separated flow rate to the total liquid flow rate increases with the gas volume flux $J_{G}$ and does not depend on the liquid volume flux $J_{L}$ so much because a large centrifugal force caused by the swirler makes most of droplets in the gas core deposit on the liquid film before the separation.

(3) In a non-swirling annular flow, $W_{s}{ }^{*}$ decreases with $J_{L}$ because of the increase in the droplet flow rate caused by the enhancement of entrainment.

(4) A local peak appears in the axial distribution of film thickness. The peak position corresponds to the location where the droplet deposition caused by the centrifugal force has completed. The position of a pick-off-ring for separating liquid should be determined so as to avoid the peak position because the performance of separation is to be low if the maximum film thickness is larger than the size of the gap between the barrel and the pick-off-ring.

(5) All the measured data will be of great use for modelling and verification of numerical methods for predicting two-phase flows in a steam separator.

\section{Acknowledgements}

The authors gratefully acknowledge the assistance in experiments by Mr. Daisuke Nishiwaki and the financial support provided by "Innovation and Viable Nuclear Energy Technology Development Project”, Ministry of Economy, Trade and Industry, Japan.

\section{References}

(1) Wakasugi, K., Nakanishi, T., Sakai, S., Wakai, K. and Sumida, I., Properties of Gas Liquid Two Phase Swirling Flow in a Vertical Long Tube, Transactions of the Japan Society of Mechanical Engineers, Series B, Vol. 60, No. 578 (1994), pp. 3345-3351.

(2) Yoneda, K. and Inada, H., Flow Characteristics of Centrifugal Gas-Liquid Separator, 
Transactions of the Japan Society of Mechanical Engineers, Series B, Vol. 70, No. 690 (2004), pp. 363-370.

(3) Nakao, T., Murase, M., Ishida, N., Kawamura, T., Minato, A. and Moriya, K., Decreasing of Pressure Loss in BWR Steam Separator (1) (Evaluation Method of Gas-Liquid Separation), Japanese Journal of Multiphase Flow, Vol. 15, No. 4 (2001), pp. 382-389.

(4) Ikeda, H., Shimizu, T., Narabayashi, T., Kondo, T., Nishida, K. and Fukuda, T., Improvement of BWR Steam Separator with Three-dimensional Gas-Liquid Two-Phase Flow Simulation Method, on CD-ROM of 11th International Conference on Nuclear Engineering (ICONE-11), Paper No. 36486 (2003), pp. 1-9.

(5) Takamasa, T. and Hazuku, T., Measuring a Film Flowing Down a Vertical Wall Using Laser Focus Displacement Meters (1st Report, Measuring Principle and Film Thickness), Transactions of the Japan Society of Mechanical Engineers, Series B, Vol. 64, No. 617 (1998), pp. 128-135.

(6) Hills, J. H., Azzopardi, B. J. and Barhey, A. S., Spatial Unsteadiness -A Way Towards Intensive Gas-Liquid Reactors-, Transactions of the Institution of Chemical Engineers, Vol. 74, Part A (1996), pp. 567-574.

(7) Mishima, K. and Ishii, M., Flow Regime Transition Criteria for Upward Two-Phase Flow in Vertical Tubes, International Journal of Heat Mass Transfer, Vol. 27, No. 5 (1984), pp. 723-737.

(8) Fukano, T., Ishida, K., Nomura, H., Akenaga, H, Odawara, H. and Takamatsu, Y., Liquid Films Flowing Concurrently with Air in Horizontal Duct (2nd Rep., Characteristics of Two-Dimensional Wave and Disturbance Wave Regions), Transactions of the Japan Society of Mechanical Engineers, Series B, Vol. 46, No. 409 (2004), pp. 1617-1625.

(9) Wallis, G. B., One Dimensional Two-Phase Flow, McGraw-Hill (1969), pp. 318-322. 